\title{
Quality of medical care begins with quality of medical education
}

\author{
Karina Marianne D. Torralba ${ }^{1}$. James D. Katz ${ }^{2}$ \\ Published online: 4 January 2020 \\ (C) International League of Associations for Rheumatology (ILAR) 2020
}

The tradition of teaching conjures images of teachers making presentations in the classroom [1]. In essence, traditional education is indoctrination in the literal sense. Put another way, the instructor-centered model of education is one wherein professors present information, as if a gift —or present - to students. Indeed, apropos to the arena of medical education, we commonly speak of "presenting a case." Unfortunately, the adult learner may not always be receptive to such didactics. This may be because the student does not feel connected (or "hooked in") to the material. Specifically, the material may not be germane to the pragmatic needs of the trainee. Or, alternatively, it may not suit the timing of professional development wherein the learner resides.

In contrast, a learner-centered experience is the modern mantra of the new academics [2]. The learner-centered experience is one wherein the lesson begins with the student. It asks, "Who is the learner?"; "What is their current knowledge?"; and "What does the student need to know?" From this scaffold, the teacher may better function as a team leader, better identify learning objective norms, and appropriately tailor mechanism to achieve professional development. The end result of learner-centered teaching is that learners will be in a position to gain a sense of ownership of their education [3]. In a learner-centered environment, students discover for themselves, they self-regulate and are accountable for their learning. They practice under supervision and enjoy the opportunity to reflect and self-assess. It behooves

Part of the Topical Collection on "Empowering Medical Education to Transform: Learnings from an international perspective"

Karina Marianne D. Torralba

KTorralba@1lu.edu

1 Department of Medicine, Division of Rheumatology, Loma Linda University School of Medicine, 11234 Anderson St, MC 1519, Loma Linda, CA 92373, USA

2 National Institutes of Health/NIAMS, NIAMS Rheumatology Fellowship and Training Branch, 10N-311A; Building 10 9000, Rockville Pike, Bethesda, MD 20892, USA those of us functioning as teachers to remember that meaningful learning is not just what people know but also how they come to know it.

The Institute of Medicine (IOM) has published Aims for Improvement in patient care [4]. The six aims for improvement include making health care delivery safe, effective, patient-centered, timely, efficient, and equitable. To this end, medical trainees must assess whether or not care meets these standards, equally and individually $[5,6]$. At a more global level, it is common to find health care outcomes employed as a measure of quality of care in specific relation to the implementation of management guidelines. Adherence to guidelines is also employed as surrogates of medical professionalism. However, generating data on medical outcomes and relating that to the implementation of guidelines ignores the foundation of quality care, namely, antecedent training and education initiatives. Training the next generation of physician-scientists is itself a scientific endeavor that falls squarely within the purview of the IOM Aims for Improvement.

Efforts towards bolstering strategies aimed at enhancing medical education are impactful to quality improvement. Although initiatives to address waste, delays, workarounds, and other roadblocks to quality care are frequently emphasized in this regard, it is the science of adult education, or andragogy, wherein deep transformational learning occurs. Andragogy seeks to enable trainees to demonstrate an awareness of, and responsiveness to, the larger context of health care [4]. It is situational learning that is meant to be an opportunity for learners to grow professionally within a clinical setting and for these same trainees to share knowledge of how to improve the systems that govern the care of a population of patients. Such learning may capitalize on straightforward chart reviews or clinic observations. It frequently involves the search for evidence-based medicine wherein one examines efforts that have produced desired and reproducible outcomes. Alternatively, it may emphasize assessments of cultural competence or capitalize on academic reviews of a select panel of patients. Ultimately, in all these situations, the trainee is called 
upon to reflect upon the learning achieved and to answer the question, "What was learned and what needs to be improved?"

A broad approach to medical education must not overlook the clinical learning environment. Among other issues, this includes identifying resource and access limitations, communication, policies, workload, and distraction. For example, human factors (e.g., problem solving strategies), system design factors (e.g., incorporating a sociocultural approach to learning), and communities of practice (e.g., clinical anatomy seminars) represent mechanisms that serve to integrate so-called "book learning" with real-world adult development. Specifically, such learning activities address the organizational culture including its receptiveness to critique and change, the need to improve collaborative communication between health care providers, and the performance of the individual in relationship to the group.

In the clinical environment, adult learning is a shared activity. It seeks to discover changes that are sustainable. This is first and foremost a mechanism for self-reflection and selfimprovement. It builds upon an individual's prior knowledge and skills allowing for the incorporation of new attitudes or more advanced concepts. It operationalizes the oft-repeated "life-long learning" aim of adult education. At the same time, it offers an opportunity for the reassessment of the definition of quality of care beyond that of solipsistically following a guideline. Learner-centered education in the clinical arena moves a community towards customizing parameters of quality of care in relation to what is relevant and applicable to their own respective health system and patient population. The IOM document explicitly states that "The fact that more than 40 percent of people with chronic conditions have more than one such condition argues strongly for more sophisticated mechanisms to coordinate care" [4]. The need for interdisciplinary care, coupled with the intrinsic complexity of rheumatic diseases, all in the setting of widespread workforce shortages, are an urgent call for graduate medical curricular reform. For rheumatologists engaged in life-long learning, and for trainees newly entering rheumatology as a field, the new academics have arrived. Early exposure, strong mentorship, community-based learning, and novel interprofessional educational engagement are interventions that will improve the visibility of, and care of, individuals with rheumatic and musculoskeletal diseases.

In summary, it is our hope that this issue of Clinical Rheumatology helps pave the way for advancing rheumatology. The articles herein may serve as a template in the aim to move rheumatology forward from a "community of practice," to "Rheumatology: a Community of Learning".

\section{References}

1. Karimi R (2011) Interface between problem-based learning and a learner-centered paradigm. Adv Med Educ Pract 2:117-125. https://doi.org/10.2147/AMEP.S12794

2. Sandars J, Cleary TJ (2011) Self-regulation theory: application to medical education: AMEE guide no 58. Med Teach 33:875-886. https://doi.org/10.3109/0142159X.2011.595434

3. Matsuyama Y, Nakaya M, Okazaki H, Lebowitz AJ, Leppink J, van der Vleuten C (2019) Does changing from a teacher-centered to a learner-centered context promote self-regulated learning: a qualitative study in a Japanese undergraduate setting. BMC Med Educ 19(1):152-112. https://doi.org/10.1186/s12909-019-1550-x

4. National Academies of Science, Engineering and Medicine. Crossing the quality chasm: a new health system for the 21 st century. http://www.nationalacademies.org/hmd/Reports/2001/Crossing-theQuality-Chasm-A-New-Health-System-for-the-21st-Century.aspx. Accessed 18 Nov 2019

5. Quinn DC, Bingham JW, Garriss GW, Dozier EA (2019) Residents learn to improve care using the ACGME core competencies and Institute of Medicine Aims for improvement: the health care matrix. J Grad Med Educ 1(1):119-126

6. Oyler J, Vinci L, Arora V, Johnson J (2008) Teaching internal medicine residents quality improvement techniques using the ABIM's practice improvement modules. J Gen Intern Med 23(7):927-930

Publisher's note Springer Nature remains neutral with regard to jurisdictional claims in published maps and institutional affiliations. 